\title{
Preliminary identification of potential PDZ-domain proteins downstream of ephrin B2 during osteoclast differentiation of RAW264.7 cells
}

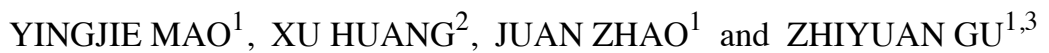 \\ ${ }^{1}$ The Affiliated Hospital of Stomatology, Medical College of Zhejiang University, 395 Yan'an Road; \\ ${ }^{2}$ The First Affiliated Hospital of Medical College of Zhejiang University, 79 Qingchun Road; \\ ${ }^{3}$ Zhejiang Chinese Medical University, 548 Bingwen Road, Hangzhou 310006, P.R. China
}

Received November 22, 2010; Accepted January 17, 2011

DOI: $10.3892 / \mathrm{ijmm} .2011 .639$

\begin{abstract}
The EphB4 receptor and ephrin B2 ligand were recently reported to influence the coupling between osteoclasts and osteoblasts in bone biology, but their downstream signaling pathways remain unclear. This study focuses on the preliminary identification of downstream PDZ-domain proteins involved in EphB4/ephrin B2 reverse signaling in osteoclasts. Similarly to primary osteoclast precursors isolated from the bone, we observed that the RAW264.7 cell line, a mouse monocyte/macrophage cell line that is used in conventional assays for osteoclast function, expressed ephrin B2 during RANKL-induced osteoclast differentiation, and that preclustered EphB4 inhibited this osteoclast differentiation. The results demonstrate that RAW264.7 cells provide a good model for further research of EphB4/ ephrin B2 signaling in osteoclasts. Immunofluorescence staining and Western blot analysis revealed that all of the eight PDZ-domain proteins previously reported to interact with ephrin B ligands were expressed in the differentiated RAW264.7 osteoclasts. However, in a co-immunoprecipitation assay, only Dishevelled 2 (Dvl2) among eight PDZ-domain proteins tested co-precipitated with ephrin $\mathrm{B} 2$ and vice versa, suggesting an endogenous interaction between Dvl2 and ephrin B2 in RANKL-induced osteoclasts. Furthermore, preclustered EphB4 reduced the expression level of Dvl2. Collectively, our results indicate that Dvl2 could be the potential PDZ-domain protein that acts downstream of ephrin B2 in RANKL-induced osteoclast differentiation of RAW264.7 cells, providing a potential novel therapeutic target for bone diseases.
\end{abstract}

Correspondence to: Dr Zhiyuan Gu, The Affiliated Hospital of Stomatology, Medical College of Zhejiang University, 395 Yan'an Road, Hangzhou 310006, P.R. China

E-mail: gzy@zju.edu.cn

Key words: EphB4, ephrin B2, receptor activator of NF-кB ligand, osteoclastogenesis, Dishevelled 2

\section{Introduction}

Bone remodeling is a complex process requiring 'coupling' between osteoclastic bone resorption and osteoblastic bone formation (1). This coupling is of great importance because a large majority of bone diseases that result in changes in bone mass, such as osteoporosis and osteopetrosis, are due to disruption of the coupling (2). Osteoclasts, large, multi-nucleated, terminally differentiated cells, are produced by the fusion of osteoclast precursor cells, while osteoblasts are derived from mesenchymal cells (3). The molecular mechanisms that are responsible for osteoclast-osteoblast communication remain one of the central issues in bone cell biology. Over the past decades, the pivotal roles of the receptor activator of $\mathrm{NF}-\kappa \mathrm{B}$ (RANK) (4), its ligand RANKL (4-6), and the natural decoy receptor for RANKL, osteoprotegerin (OPG) (7-9) have been well-documented. The binding of RANKL, which is released by osteoblasts and bone marrow cells, to its receptor RANK has an essential role in the differentiation and fusion of precursors into mature osteoclasts $(10,11)$.

There is recent evidence to suggest a role for the Eph receptors and their ephrin ligands in bone biology, a role that may affect the coupling between osteoblasts and osteoclasts $(12,13)$. The Eph receptors are the largest subgroup of the receptor tyrosine kinase family and interact with ephrin ligands. Fourteen Eph receptors (EphA1-A8 and EphB1-B6) and eight ephrin ligands (ephrin A1-A5 and ephrin B1-B3) have been identified in the human genome (14). Zhao et al (12) were the first to report on EphB4/ephrin B2 signaling in bone remodeling. Briefly, ephrin B2-expressing osteoclasts stimulated the differentiation of EphB4-expressing osteoblasts via forward signaling, and reverse signaling through ephrin B2 inhibited the differentiation of osteoclasts. This bidirectional signaling may have a pivotal role in the transitional phase of bone remodeling and in the promotion of osteoblast differentiation by osteoclasts and concurrent suppression of osteoclast differentiation by osteoblasts $(2,12)$. Following the first report, there was new evidence about the involvement of Eph/ephrin in bone remodeling. For example, PTH and PTHrp can elevate the expression level of ephrin B2 in osteoblasts, indicating a cell-mediated paracrine mode of EphB4/ephrin B2 
communication within the osteoblast lineages (15). However, the downstream element involved in EphB4/ephrin B2 reverse signaling in osteoclasts has not yet been identified. As known from a previous review, reverse signaling through ephrin B ligands can activate both tyrosine phosphorylation-dependent and -independent signal transduction pathways. The intracellular domain of ephrin B ligands, particularly the last 33 C-terminal amino acids, is highly conserved and contains multiple tyrosine residues, and the C-terminal YKV motif is a binding site for PDZ (post-synaptic density protein, disks large, zona occludens) domain-containing proteins. Additionally, the data of Zhao et al showed that in osteoclasts, the reverse inhibitory effect was dependent on the cytoplasmic domain of ephrin B2, while the C-terminal YKV motif was critical for signal transduction, indicating that an unknown downstream PDZ-domain protein was involved (12).

This pilot study was designed to confirm the effect of bidirectional EphB4/ephrin B2 signaling on RANKL-induced osteoclastogenesis, to verify whether RANKL-stimulated RAW264.7 cells could be substituted for primary cells as a model for research on the EphB4/ephrin B2 signaling pathways, and most importantly, to preliminarily identify the potential PDZ-domain proteins acting downstream of ephrin B2 during osteoclast differentiation of RAW264.7 cells in vitro.

\section{Materials and methods}

Materials. All media components were purchased from Gibco (Invitrogen Corp., Carlsbad, CA). The RNA extraction reagent TRIzol was from Invitrogen Life Technologies. The leukocyte acid phosphatase kit (no. 387) was from Sigma Chemical Co. (St. Louis, MO). The goat anti-human IgG-Fc $\gamma$ fragment-specific antibody and the human $\mathrm{IgG}-\mathrm{Fc}$ fragment were from Jackson ImmunoResearch Laboratories, Inc. (West Grove, PA). Recombinant murine sRANKL and recombinant murine EphB4-Fc chimera were obtained from R\&D Systems, Inc. (Minneapolis, MN). Goat polyclonal antibodies against ephrin B2 (H-83), GRIP2 (K-17), and RGS3 (H-300) and rabbit polyclonal antibodies against GRIP1 (R-91), FAP-1 (H-300) and $\beta$-actin were from Santa Cruz Biotechnology (Santa Cruz, CA). Rabbit polyclonal antibodies against Dishevelled 2 (Dvl2, ab22616), PICK1 (ab3420) and Syntenin (ab19903) were from Abcam, Inc. (Cambridge, MA). The rabbit polyclonal antibody against PAR3 (07-330) was from Upstate Biotechnology, Inc. (Charlottesville, VA). TRITC/FITC-conjugated rabbit anti-goat and goat anti-rabbit $\mathrm{IgG}(\mathrm{H}+\mathrm{L})$ antibodies were from PeproTech, Inc. (Rocky Hill, NJ). The PrimeScript ${ }^{\mathrm{TM}}$ RT reagent kit and the $\mathrm{SYBR}^{\circledR}$ Premix Ex Taq ${ }^{\mathrm{TM}}$ kit were from Takara Biotechnology Co., Ltd. (Dalian, China). Protein A+G agarose was from Beyotime Biotech (Haimen, China).

Cell cultures. The RAW264.7 mouse monocyte/macrophage cell line was purchased from the American Type Culture Collection (ATCC, TIB-71). RAW264.7 cells were maintained in Dulbecco's modified Eagle's medium (DMEM) containing $10 \%$ heat-inactivated fetal bovine serum (FBS). All media were supplemented with $2 \mathrm{mM}$ glutamine, $100 \mathrm{IU} / \mathrm{ml}$ penicillin and $100 \mu \mathrm{g} / \mathrm{ml}$ streptomycin (Sigma). Incubations were performed at $37^{\circ} \mathrm{C}$ with $5 \% \mathrm{CO}_{2}$ in humidified air.
Tartrate-resistant acid phosphatase (TRAP) staining. RAW264.7 cells were suspended in DMEM supplemented with $1-2 \%$ FBS and seeded at a concentration of $1 \times 10^{4}$ cells/well into a 24-well culture plate (Corning) with pre-set sterilized slides. After $12 \mathrm{~h}$ of starvation, the media were replaced with phenol red-free $\alpha$-MEM supplemented with $10 \%$ FBS and $25 \mathrm{ng} / \mathrm{ml}$ RANKL with the addition of $2 \mu \mathrm{g} / \mathrm{ml}$ EphB4-Fc or Fc fragments as a negative control. Before application to the cultured cells, EphB4-Fc or Fc fragments were preclustered with anti-human $\mathrm{Fc}$ antibody at a $1: 10$ molar ratio at $4^{\circ} \mathrm{C}$ for $1 \mathrm{~h}$. The media were replaced every two days. After four days of culture, the cells were subjected to TRAP staining by a leukocyte acid phosphatase kit according to the manufacturer's instructions. Briefly, the cells were washed twice in PBS and fixed for $30 \mathrm{sec}$ in a solution of $4 \%$ formaldehyde combined with a citrate solution and acetone. Then, the fixed cells were washed in PBS and incubated in TRAP staining solution for $1 \mathrm{~h}$ at $37^{\circ} \mathrm{C}$. After the TRAP reaction, the slides were rinsed in deionized water, air-dried and evaluated microscopically under a $\mathrm{x} 4$ objective on an Olympus IX71 inverted research microscope. Eight fields of a single well were selected randomly, and three wells were counted for each group. Multinucleated (with three or more nuclei) TRAP-positive cells (MNCs) appeared dark red and were counted as differentiated osteoclasts.

RNA extraction and cDNA synthesis. RAW264.7 cells were suspended in DMEM supplemented with 1-2\% FBS and seeded at a concentration of $5 \times 10^{4}$ cells/well into a 6 -well culture plate (Corning). After $12 \mathrm{~h}$ of starvation, the media were replaced with phenol red-free $\alpha$-MEM supplemented with $10 \%$ FBS and $25 \mathrm{ng} / \mathrm{ml}$ RANKL with the addition of $2 \mu \mathrm{g} / \mathrm{ml}$ preclustered EphB4-Fc or Fc fragments. The media were replaced every 2 days. After 4 days of culture, total RNA was extracted using TRIzol according to the manufacturer's instructions. Isolated total-RNA was eluted in RNase-free water and stored at $-80^{\circ} \mathrm{C}$ until use. cDNA reverse transcription was performed using the PrimeScript ${ }^{\mathrm{TM}}$ RT reagent kit according to the manufacturer's instructions. Briefly, $0.5 \mu \mathrm{g}$ of total RNA was mixed with $2 \mu \mathrm{l}$ of the $5 \mathrm{X}$ PrimeScript ${ }^{\mathrm{TM}}$ buffer, $0.5 \mu \mathrm{l}$ of the PrimeScript ${ }^{\mathrm{TM}}$ RT enzyme mix I, $0.5 \mu \mathrm{l}$ of random hexamer primers $(100 \mu \mathrm{M}), 0.5 \mu \mathrm{l}$ of the oligo(dt) primer $(50 \mu \mathrm{M})$ and RNase-free water to a total volume of $10 \mu \mathrm{l}$. The mixture was incubated at $37^{\circ} \mathrm{C}$ for $15 \mathrm{~min}$, and the reaction was stopped by heating at $85^{\circ} \mathrm{C}$ for $5 \mathrm{sec}$.

Quantitative real-time RT-PCR. Quantitative real-time RT-PCR was performed using the SYBR-Premix Ex Taq ${ }^{\mathrm{TM}}$ kit according to the manufacturer's instructions. The following primers were used: ephrin B2: sense, 5'-TCTGTGTGGAAGTACTGT TGGGGACTTT-3' and antisense, 5'-TGTACCAGCTTCTAG CTCTGGACGTCTT-3'; Nfatc1: sense, 5'-TGCTCCTCCTCC TGCTGCTC-3' and antisense, 5'-CGTCTTCCACCTCCAC GTCG-3'; c-Fos: sense, 5'-TCCGTCTCTAGTGCCAACTT-3' and antisense, 5'-CGCTTGGAGTGTATCTGTCA-3'; c-Jun: sense, 5'-ACTCGGACCTTCTCACGTCG-3' and antisense, 5'-TAGACCGGAGGCTCACTGTG-3'; $\beta$-actin: sense, 5 '-AGG AGCAATGATCTTGATCTT-3' and antisense, 5'-TGCCAAC ACAGTGCTGTCT-3'; TRAP (Acp5): sense, 5'-GGAAATG GCCAATGCCAAAG-3' and antisense, 5'-ATCATGGTTTC 
CAGCCAGCAC-3'; cathepsin K (Ctsk): sense, 5'-CAGCAGA ACGGAGGCATTGA-3' and antisense, 5'-CTTTGCCGTGG CGTTATACATACA-3'; calcitonin receptor (Calcr): sense, 5'-TTACCGACGAGCAACGCCTAC-3' and antisense, 5'-AGC AAGTGGGTTTCTGCACTCA-3'; Dishevelled 2 (Dvl2): sense, 5'-GCAGTGTCACCGATTCCACAA-3' and antisense, 5'-CTTTCATGATGGAGCCGATGTAGA-3'.

PCR was performed with a 4-min initial denaturation at $95^{\circ} \mathrm{C}$, followed by 40 cycles of $30 \mathrm{sec}$ of denaturation at $95^{\circ} \mathrm{C}$, $30 \mathrm{sec}$ of annealing at $60^{\circ} \mathrm{C}$, and $1 \mathrm{~min}$ of extension at $72^{\circ} \mathrm{C}$ using the Bio-Rad CFX96 system. $\beta$-actin was used as an internal control to normalize the expression of target genes. The data obtained from three independent experiments were used to analyze the relative gene expression by the $2^{-\Delta \Delta \mathrm{Ct}}$ method.

Immunofluorescence staining. RAW264.7 cells cultured on coverslips under the described conditions were fixed with methanol for $30 \mathrm{~min}$, washed in PBS, permeabilized with $0.2 \%$ Triton X-100 in PBS for 5 min, washed in PBS, and blocked with $5 \%$ bovine serum albumin (BSA) in PBS for 1-2 $\mathrm{h}$ at room temperature. After blocking, the cells were incubated overnight at $4^{\circ} \mathrm{C}$ with the specific primary antibodies at a 1:50 dilution in a 1\% BSA solution. Staining was completed with the corresponding secondary antibodies at 1:200 dilutions in a 1\% BSA solution. Then, the coverslips were mounted on slides and examined under a Nikon Eclipse 80i fluorescence microscope.

Western blot analysis. Cells collected from the described osteoclastogenic cultures were lysed in ice-cold lysis buffer [50 mM Tris (pH 7.4), $150 \mathrm{mM} \mathrm{NaCl}, 1 \%$ NP-40, $2.5 \mathrm{mM}$ sodium pyrophosphate, $1 \mathrm{mM}$ EDTA, $1 \% \mathrm{Na}_{3} \mathrm{VO}_{4}, 0.5 \mu \mathrm{g} / \mathrm{ml}$ leupeptin, sodium fluoride, $1 \mathrm{mM}$ phenylmethane sulfonyl fluoride (PMSF)]. Following centrifugation at $12,000 \mathrm{x} \mathrm{g}$ for $5 \mathrm{~min}$ at $4^{\circ} \mathrm{C}$, the supernatants were collected, and the protein concentrations were measured by a BCA kit (Pierce). Equal amounts of proteins (30 $\mu \mathrm{g} /$ lane) were run on $8-12 \%$ SDS-PAGE gels and electrophoretically transferred onto PVDF membranes (Sigma). After blocking, the membranes were probed with specific primary antibodies to detect the proteins of interest followed by the corresponding secondary antibodies. $\beta$-actin was used as an internal standard for protein concentration and integrity. Bound antibodies were visualized by the enhanced chemiluminescence substrate (Beyotime). The bands of the immunoblots were quantified by densitometry and normalized with $\beta$-actin using the BandScan software. Protein expression is expressed as a percentage of the normal control.

Co-immunoprecipitation. The cells collected from the described osteoclastogenic cultures were lysed as described above. After centrifugation, the collected supernatants were incubated with the specific primary antibody (diluted 1:50) at $4^{\circ} \mathrm{C}$ overnight. Next, protein $\mathrm{A}+\mathrm{G}$ agarose beads were added to the mixture, and the mixture was incubated at $4^{\circ} \mathrm{C}$ for $3 \mathrm{~h}$ with mixing. After centrifugation at $12,000 \mathrm{x} \mathrm{g}$ for $5 \mathrm{~min}$ at $4^{\circ} \mathrm{C}$, the beads with the bound proteins were washed five times with lysis buffer. The released proteins were resuspended in $1 \mathrm{X}$ electrophoresis sample buffer and were analyzed by Western blotting as described above.
Statistical analysis. The results are expressed as means \pm SDs from triplicate independent experiments. Data were statistically analyzed using one-way analysis of variance followed by Tukey's post-hoc test or unpaired Student's t-tests, and statistical significance was set at $\mathrm{P}$-values $<0.05$.

\section{Results}

Expression of ephrin B2 during RANKL-induced osteoclast differentiation of RAW264.7 cells. To investigate whether ephrin B2 is prominently expressed in RANKL-induced osteoclasts, we first used real-time RT-PCR to examine the mRNA levels of ephrin B2. RAW264.7 cells cultured in the presence of RANKL for four days were designated as the RANKL ${ }^{+}$ group (marked in the figures), while the cells cultured in the absence of RANKL for four days were designated as the RANKL group. The results show that the mRNA levels of ephrin $\mathrm{B} 2$ are significantly higher in the $\mathrm{RANKL}^{+}$group compared to the RANKL ${ }^{-}$group (Fig. 1A). Using Western blotting and immunofluorescence staining, the expression of ephrin B2 protein was also detected in the $\mathrm{RANKL}^{+}$group (Fig. 1B and C). Collectively, the data demonstrate that ephrin B2 is prominently expressed during RANKL-induced osteoclast differentiation of RAW264.7 cells.

EphB4/ephrin B2 signaling inhibits RANKL-induced osteoclast differentiation of RAW264.7 cells. To analyze the effects of reverse signaling on osteoclast differentiation, we used soluble EphB4-Fc to stimulate ephrin B2 because EphB4 exclusively interacts with ephrin B2 (16,17). RAW264.7 cells were cultured in the presence of RANKL with the addition of preclustered soluble EphB4-Fc or Fc, and these samples were designated as the EphB4-Fc- or the Fc-treated groups, respectively. After four days in the conditioning culture, TRAP staining was performed (Fig. 2A), and TRAP-positive MNCs in each well were counted as osteoclasts. Fig. 2B shows that the number of TRAP-positive MNCs was significantly lower in the EphB4-Fc-treated group than that in the Fc-treated group. Meanwhile, no significant difference in the number of TRAP-positive MNCs was detected between the Fc-treated group and the RANKL ${ }^{+}$group (Fig. 2A and B). The Fc-treated group was included to rule out the interference of Fc fragments and anti-Fc antibodies. Next, because differentiated osteoclasts were reported to express high levels of osteoclast-specific genes such as TRAP, Ctsk, and Calcr $(18,19)$, we used real-time RT-PCR to investigate whether the inhibitory effect of EphB4-ephrin B2 reverse signaling correlated with the mRNA levels of the osteoclast-specific genes. After four days in the conditioning culture, total RNA was collected and analyzed, and the results showed that the EphB4-Fc treatment reduced the mRNA expression levels of TRAP, Ctsk and Calcr compared with the Fc treatment (Fig. 2C). These data demonstrate that the addition of preclustered soluble EphB4-Fc to the osteoclastogenic cultures inhibits the RANKL-induced osteoclast differentiation of RAW264.7 cells.

EphB4/ephrin B2 signaling suppresses the expression of c-Fos, c-Jun and Nfatcl in RANKL-stimulated RAW264.7 cells. The Nfatc1 gene has been identified as the most strongly 
A

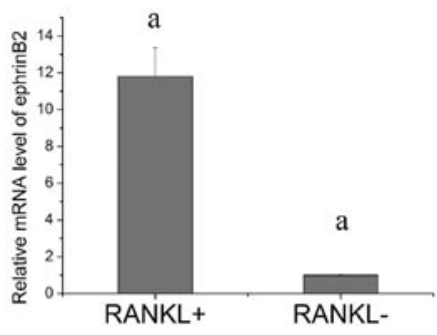

B

RANKL $+\quad-$

ephrin B2

Actin
$\mathrm{C}$

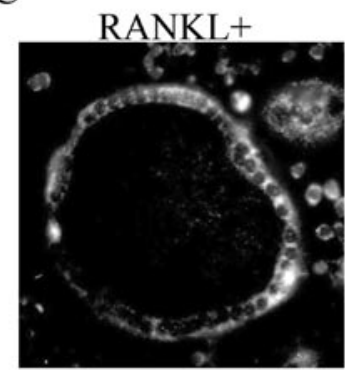

RANKL-

Figure 1. Expression of ephrin B2 was prominently elevated after osteoclast differentiation of RAW264.7 cells in the presence of RANKL for four days, compared with undifferentiated RAW264.7 cells cultured in the absence of RANKL. (A) Real-time RT-PCR analysis of ephrin B2 gene expression. $\beta$-actin was used as an internal control to normalize the expression of the target genes. Bars represent the means \pm SDs. The data are representative of three different experiments. RANKL ${ }^{+}$group vs. RANKL group; ${ }^{a} \mathrm{P}<0.01$. (B) Immunoblot analysis of ephrin $\mathrm{B} 2$ protein. Actin served as a loading control. (C) Immunofluorescence of ephrin $\mathrm{B} 2$ protein; magnification $\mathrm{x} 400$.

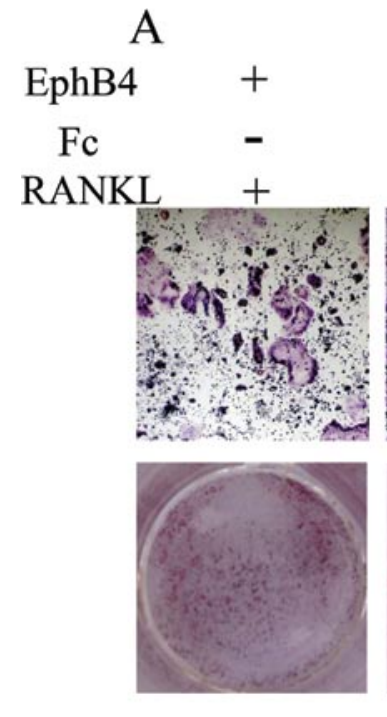

B

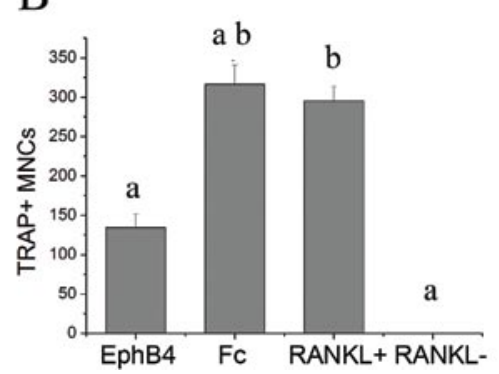

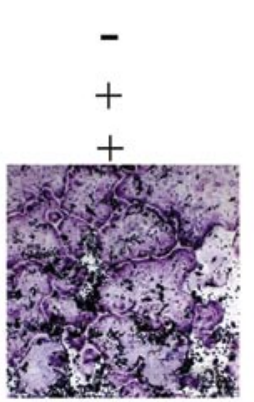
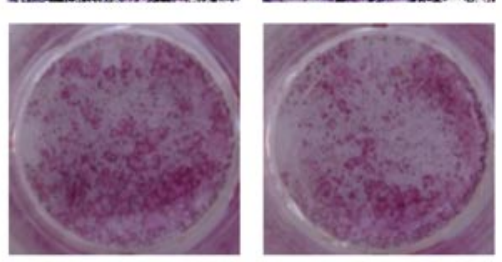

$\mathrm{C}$

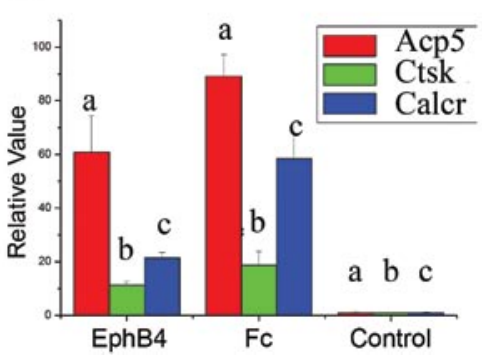

Figure 2. EphB4-ephrin B2 reverse signaling suppresses the RANKL-induced osteoclast differentiation of RAW264.7 cells. (A) TRAP staining of differentiating and differentiated osteoclasts derived from RANKL-stimulated RAW264.7 cells in the presence of EphB4-Fc or Fc fragments ( $2 \mu \mathrm{g} / \mathrm{ml})$ for four days; magnification x100. (B) Quantitative analysis of TRAP-positive multinuclear cells with three or more nuclei in each well. EphB4 group vs. the Fc group and the RANKL group, ${ }^{\mathrm{a}} \mathrm{P}<0.01$; Fc group vs. the RANKL ${ }^{+}$group, ${ }^{\mathrm{b}} \mathrm{P}>0.05$. (C) Real-time RT-PCR analysis of TRAP (Acp5), calcitonin receptor (Calcr) and cathepsin K (Ctsk) after osteoclast differentiation of RANKL-stimulated RAW264.7 cells in the presence of EphB4-Fc or Fc fragments ( $2 \mu \mathrm{g} / \mathrm{ml})$ for four days. The cells cultured without RANKL or EphB4-Fc or Fc were used as the control in this and in the following experiments. $\beta$-actin was used as an internal control to normalize the expression of target genes. Bars represent the means \pm SDs. All data are representative of three independent experiments. EphB4 group vs. Fc group and control group; ${ }^{\mathrm{a}} \mathrm{P}<0.01,{ }^{\mathrm{b}} \mathrm{P}<0.01$ and ${ }^{\mathrm{c}} \mathrm{P}<0.01$. 
A

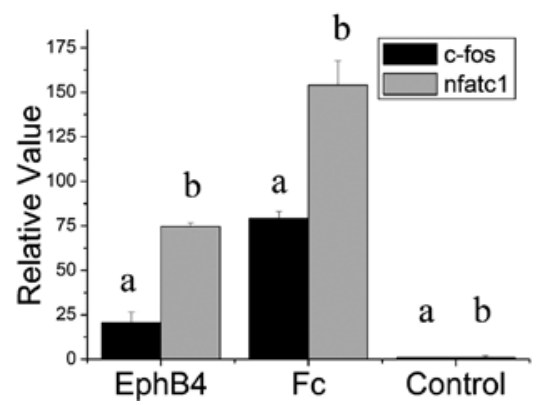

B

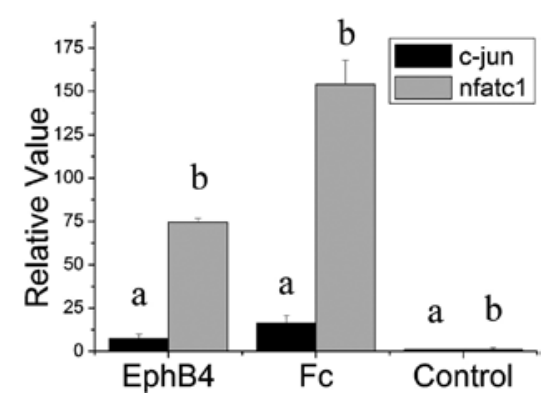

Figure 3. Effect of EphB4-ephrin B reverse signaling on the expression of c-Fos, c-Jun and Nfatc1 during RANKL-induced osteoclast differentiation of RAW264.7 cells. (A) Real-time RT-PCR analysis of c-Fos and Nfatcl gene expression. $\beta$-actin was used as an internal control to normalize the expression of target genes. Bars represent the means \pm SDs. All data are representative of three independent experiments. EphB 4 group vs. Fc group and control group ${ }^{a} \mathrm{P}<0.01$ and ${ }^{b} \mathrm{P}<0.01$. (B) Real-time RT-PCR analysis of c-Jun and Nfatc1 gene expression. $\beta$-actin was used as an internal control to normalize the expression of target genes. Bars represent the means \pm SDs. The data are representative of three independent experiments. EphB4 group vs. Fc group and control group; ${ }^{\mathrm{a}} \mathrm{P}<0.01$ and ${ }^{\mathrm{b}} \mathrm{P}<0.01$.

A
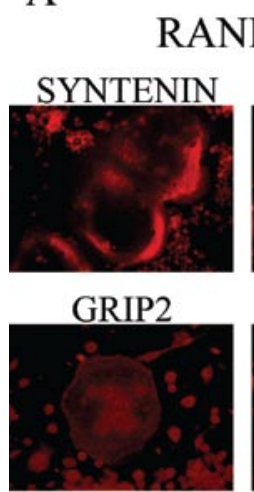

EAP-1

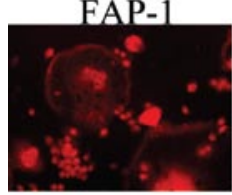

PICK1

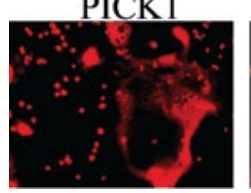

B
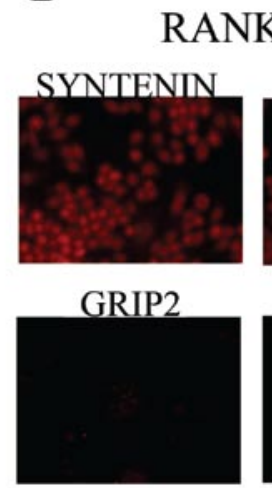

FAP-1

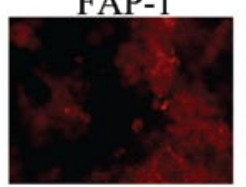

PICK1

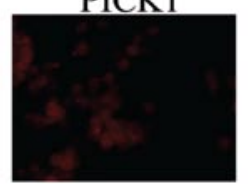

Dvl2

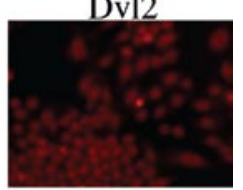

PAR3

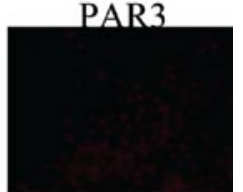

GRIP1

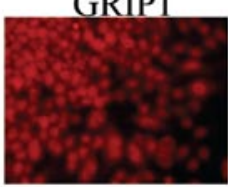

RGS3

Figure 4. Immunofluorescence of potential PDZ-domain proteins during RANKL-induced osteoclast differentiation of RAW264.7 cells. Immunofluorescence of Syntenin, Dv12, GRIP2, PAR3, FAP-1, GRIP1, PICK1 and RGS3 in RAW264.7 cells in the presence (A) or absence (B) of RANKL for four days. Magnification x100.

induced transcription factor following RANKL-stimulated osteoclast differentiation in RAW264.7 cells $(20,21)$. The osteoclastogenic activity of Nfatc1 has been shown to be enhanced by overexpression of c-Jun and c-Fos but is inhibited by overexpression of a dominant-negative form of either c-Jun or c-Fos, indicating that the partnership between c-Jun/c-Fos and Nfatc1 is crucial for osteoclast differentiation (22-24). In this study, we used real-time RT-PCR to examine whether EphB4/ephrin B2 reverse signaling modulates these critical transcription factors at the mRNA level in RANKL-induced RAW264.7 cells. The results show that the mRNA levels of these transcription factors were induced in the EphB4-Fctreated group compared to the Fc-treated group (Fig. 3).
Identification of potential PDZ-domain proteins expressed during the RANKL-induced osteoclast differentiation of RAW264.7 cells. Ephrin B2 ligands can recruit PDZ-domain proteins through a carboxy-terminal YKV motif. So far, eight PDZ-domain proteins (PDZ-RGS3, Dv12, PICK1/ PHIP, Syntenin, GRIP1, GRIP2, PTP-BL/FAP-1 and PAR3) have been described in the literature to bind ephrin $\mathrm{B}$ ligands $(14,25)$. We screened to assess whether any of the eight PDZ-domain proteins are expressed during RANKL-induced osteoclast differentiation of RAW264.7 cells. As shown in Figs. 4 and 5, the immunofluorescence staining and Western blot analysis revealed that the eight PDZ-domain proteins were all expressed in the $\mathrm{RANKL}^{+}$group after four days in 


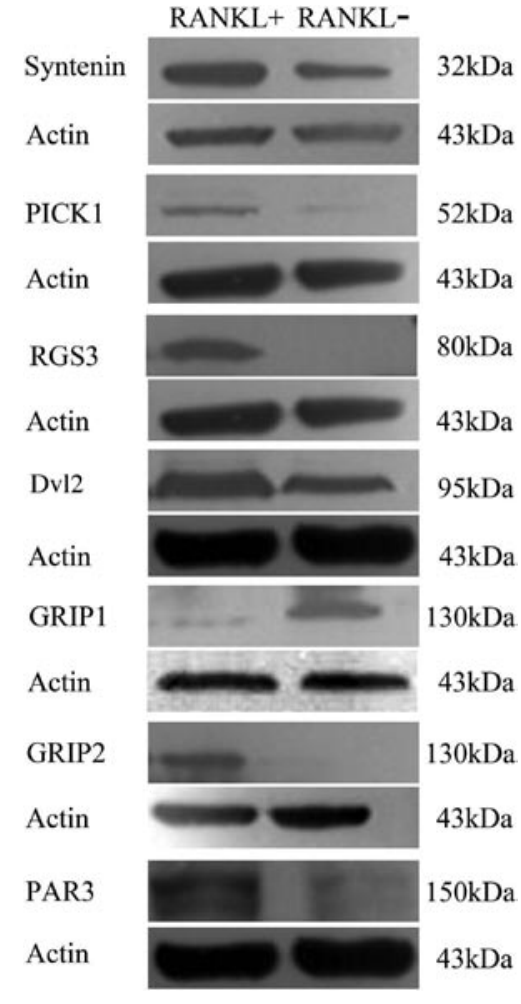

Figure 5. Immunoblot analysis of potential PDZ-domain proteins during RANKL-induced osteoclast differentiation of RAW264.7 cells. Immunoblot analysis of Syntenin, Dv12, GRIP2, PAR3, GRIP1, PICK1 and RGS3 after osteoclast differentiation of RAW264.7 cells in the presence or absence of RANKL for four days. The data for FAP-1 were not obtained because of the high molecular weight $(250 \mathrm{kDa})$ and technical problems, but this had little effect on the study. $\beta$-actin served as a loading control. All of the paired blots were obtained from one lysate and were also examined within one experiment with equal gel loading.

the conditioning culture. As a control, in the undifferentiated RAW264.7 precursors (RANKL- group), four PDZ-domain proteins (PDZ-RGS3, PICK1/PHIP, GRIP2 and PAR3) were not expressed and two PDZ-domain proteins (Dv12 and Syntenin) were expressed at a lower level (Figs. 4 and 5). Only GRIP1 was more strongly expressed in the undifferentiated RAW264.7 precursors than in the differentiated ones (Figs. 4 and 5). The data indicate that the eight PDZ-domain proteins are all expressed in the RANKL-induced osteoclasts, prompting us to include all of them in the following analysis.

Identification of potential PDZ-domain proteins as binding partners for the PDZ binding site of ephrin B2. To investigate whether ephrin B2 ligands associate with any of these eight PDZ-domain proteins, we immunoprecipitated ephrin B2 proteins from the cell lysate with a goat polyclonal antibody, and the precipitate was assayed for co-immunoprecipitated PDZ-domain proteins by immunoblotting. As shown in Fig. 6A, among the eight proteins, only Dvl2 showed the expected size co-precipitating with ephrin B2. The reverse experiment (immunoprecipitation by the Dvl2 antibody and immunoblotting by the ephrin B2 antibody) confirmed the relationship. These data indicate an interaction between endogenously expressed Dvl2 and ephrin B2 in the RANKLinduced osteoclasts. However, the other PDZ-domain proteins were not detected at the expected sizes in the precipitate after immunoprecipitation with the ephrin B2 antibody in three independent experiments. Considering the above data, we suggest that Dvl2 protein might be the potential PDZ-domain protein involved downstream of ephrin B2.

Finally, to investigate whether the EphB4-ephrin B2 reverse signaling affects the expression level of Dvl2, preclustered soluble EphB4-Fc or Fc fragments were added to the RANKLinduced osteoclastogenic cultures. After four days in the conditioning culture, the Western blot analysis showed that the band for Dvl2 in the EphB4-Fc-treated group was weaker than that in the Fc-treated group (Fig. 6B), and the mRNA level of Dvl2 in the EphB4-Fc-treated group was a little lower than in the Fc-treated group, albeit with no statistical significance (Fig. 6C). These data indicate that EphB4-ephrin B2 reverse signaling down-regulates the expression level of Dvl2.

\section{Discussion}

For more than two decades, we have known that the differentiation and activation of osteoclasts depends on signaling of the RANK receptor stimulated by RANKL. However, the role of Eph/ephrin in the coupling between osteoblasts and osteoclasts has been reported within the last few years $(12,13)$. Although the interaction between ephrins and Eph receptors in cardiovascular development, skeletal development, axon guidance and tissue patterning has been extensively investigated, there is insufficient evidence for the roles of Eph/ephrin interactions in bone biology (26). Furthermore, there is little research focusing on the potential molecular interactions of EphB4/ephrin B2 intracellular reverse signaling. In this study, we demonstrated that EphB4/ephrin B2 reverse signaling inhibits the RANKL-induced osteoclast differentiation of RAW264.7 cells and that Dvl2 may be the potential downstream PDZ-domain protein that is the binding partner for the C-terminal YKV motif of ephrin B2.

The first step of our study was to select the type of osteoclastic precursors to be used for further research. Primary bone marrow stromal cells may be a good choice according to a report by Zhao et al (12). However, inter-individual variability and insufficient material are disadvantages for the studies with primary cell cultures (27). On the other hand, cell lines are being increasingly recognized as standard experimental materials and as essential components to ensure reproducible and reliable results in life science research. RAW264.7 cells have been extensively used as osteoclast precursors because they can be induced to stably differentiate into osteoclast-like cells by using RANKL with or without M-CSF, and RAW264.7 cell-derived osteoclasts perform similarly to primary osteoclasts isolated from bones in conventional assays for osteoclast function (28). In addition, ephrin B2 mRNA was reported to be expressed in RAW264.7 cells (29). We proposed that RAW264.7 cells may be a suitable model for this research. As shown in Fig. 1C, TRAPpositive, multinucleated osteoclasts expressed ephrin B2 protein distributed in the cell membrane after four days of treatment with RANKL. The induced osteoclast differentiation of RAW264.7 cells could be suppressed by the addition of preclustered soluble EphB4-Fc to the culture (Fig. 2). Additionally, the reduction of TRAP-positive multinucleated cells with three or more nuclei was more apparent in 

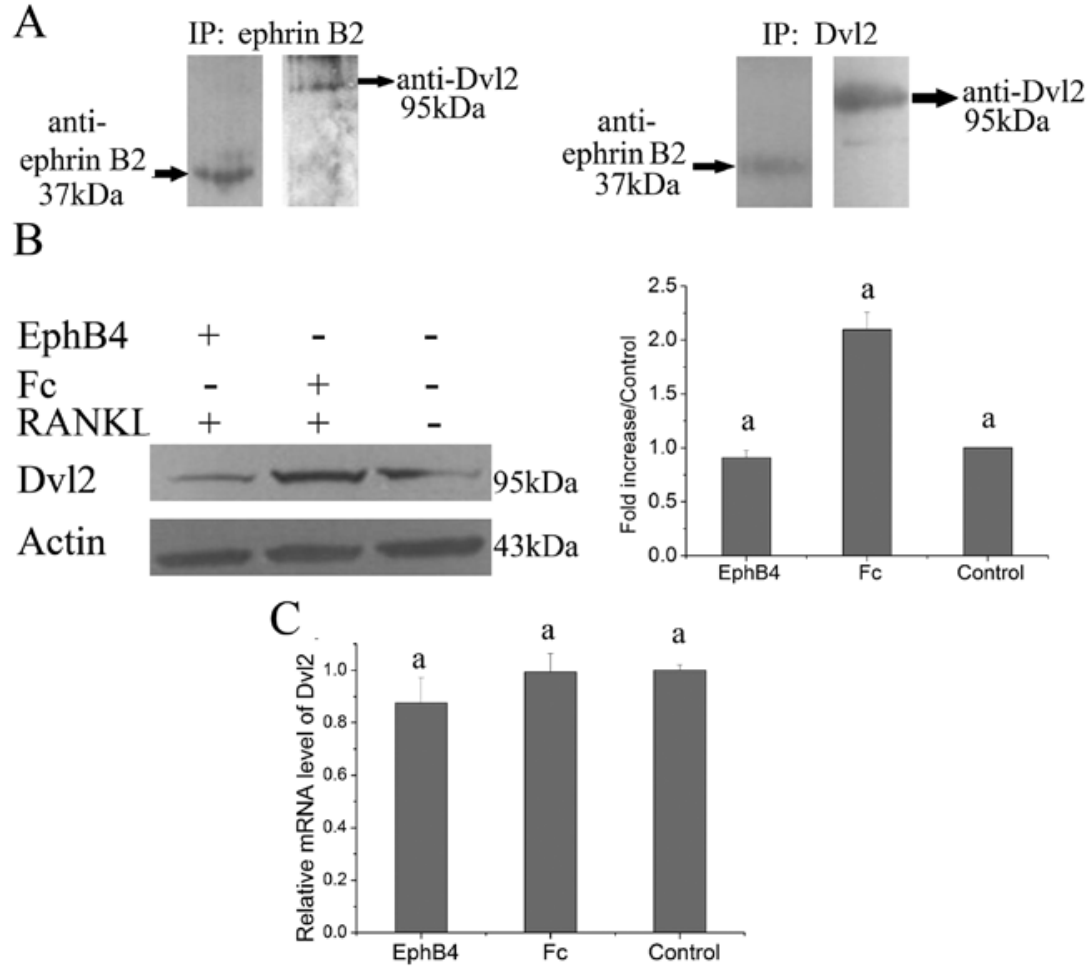

Figure 6. Identification of potential PDZ-domain proteins as binding partners for the PDZ binding site of ephrin B2. (A) We immunoprecipitated ephrin B2 protein from the cell lysis buffer with a goat polyclonal antibody against ephrin B2 (1:50) and assayed for co-immunoprecipitated PDZ-domain proteins by immunoblotting. Among the eight PDZ-domain proteins (PDZ-RGS3, Dv12, PICK1/PHIP, Syntenin, GRIP1, GRIP2, PTP-BL/FAP-1 and PAR3) expressed during RANKL-induced osteoclastogenesis of RAW264.7 cells, only Dv12 was detected by the Western blot analysis. Then, we immunoprecipitated Dv12 proteins from the cell lysis buffer, and detected ephrin B2 in the precipitate by immunoblotting. (B) Immunoblot analysis of Dv12 proteins in the EphB4-Fc treated group compared with the Fc-treated or the untreated blank control groups. Actin protein expression served as an internal control and was used to normalize the protein band intensity. The bar represents the relative Dvl2 protein levels, (percent of control group). EphB4 group vs. Fc group; ${ }^{\mathrm{P}}<0.01$. (C) Real-time RT-PCR analysis of Dv12 mRNA expression (the EphB4-Fc treatment compared with the Fc-treated or the untreated blank control group). $\beta$-actin was used as an internal control to normalize the expression of target genes. Bars represent the means \pm SDs. The data are representative of three independent experiments. EphB4 group vs. Fc group and control group; ${ }^{a}>0.05$.

the EphB4-Fc-treated group compared with the Fc-treated group after RANKL-induced osteoclast differentiation. The mRNA levels of TRAP, Cstk, and Calcr, well-known markers of osteoclast differentiation, were also down-regulated in the EphB4-Fc-treated group. From all of these data, we concluded that RAW264.7 cells could be used as osteoclast precursors for further research. Stimulation of Nfatc1, the transcriptional target of c-Jun/c-Fos, has demonstrated the necessity of Nfatc1 for the RANKL-induced osteoclastogenesis of RAW264.7 cells in vitro (23). The transcription of the c-Jun and c-Fos genes was up-regulated when osteoclast precursor cells were stimulated with RANKL (30). Ikeda et al and Matsuo et al proposed that the partnership between c-Jun/c-Fos and the NFAT family was crucial for osteoclast differentiation $(23,24)$. Regarding the importance of these transcription factors in the downstream pathways of RANKL-RANK signaling, we investigated whether EphB4/ephrin B2 reverse signaling modulated the expression of these transcription factors during RANKL-induced osteoclastogenesis. Interestingly, the mRNA levels of c-Jun, c-Fos and NFATc1 were inhibited in the EphB4-Fc-treated group during RANKL-induced osteoclast differentiation of RAW264.7 cells compared with the Fc-treated group. These results are consistent with the observed inhibition of RANKL-induced osteoclast differentiation of RAW264.7 cells by the EphB4/ephrin B2 reverse signaling mentioned above. Zhao et al claimed that EphB4/ ephrin B2 reverse signaling inhibited the osteoclastogenesis of primary osteoclast precursors partly by reducing the c-Fos/ Nfatc1 partnership (12). Our data not only confirm their conclusion in RAW264.7 cells, but also suggest the involvement of a c-Jun/Nfatc1 partnership.

Ephrin B2 ligands can recruit PDZ-domain proteins through a carboxy-terminal YKV target site. The analysis of the cytoplasmic region of ephrin B2 demonstrated that the inhibitory effect of EphB4/ephrin B2 reverse signaling in differentiated osteoclasts was mediated via an interaction with PDZ domain proteins but not by tyrosine phosphorylation, thus raising the possibility that the PDZ domain proteins might be useful drug targets in bone remodeling $(2,12)$. Therefore, we attempted to identify the candidate PDZ-domain proteins downstream of ephrin B2 during RANKL-induced osteoclast differentiation of the RAW264.7 cell line. So far, there have been eight PDZ-domain proteins reported to interact with ephrin B ligands, including PDZ-RGS3, Dv12, PICK1/ PHIP, Syntenin, GRIP1, GRIP2, PTP-BL/FAP-1 and PAR3 $(14,25)$. Using immunofluorescence staining and Western blot analysis, we noted that all of the eight PDZ-domain proteins were expressed in the differentiated RAW264.7 osteoclasts, but in the co-immunoprecipitation analysis, only Dvl2 protein co-precipitated with ephrin B2 and vice versa, indicating an 
endogenous interaction between Dvl2 proteins and ephrin B2 in the RANKL-induced osteoclasts.

Dvl2 is a member of the Dishevelled (Dsh) protein family and is a cytoplasmic protein containing three conserved protein domains: DIX (Dishevelled-Axin), PDZ and DEP. It was reported that the $\mathrm{C}$-terminus of ephrin $\mathrm{B} 1$ was necessary for a physical interaction with Dsh $(31,32)$. Xdsh, which is reported to form a complex with EphB receptors and also with ephrin B1, was involved in Eph- and ephrin-mediated cell repulsion as a downstream effector of these receptors and ligands (33). Because ephrin B1 and ephrin B2 share a high degree of identity $(12,34)$, our results suggest that the C-terminal end of ephrin B2 can interact with Dsh directly or indirectly.

Because the phosphorylation of specific tyrosine residues in the intracellular domain of ephrin B1 by the extracellular domain of the cognate Eph receptor can disrupt the inter-action of ephrin B1 with the endogenous Dsh protein (32), we propose that after binding of EphB4, the disruption of the ephrin B2/Dvl2 complex inhibits RANKL-induced osteoclast differentiation in RAW264.7 cells. The next question would be whether there is any potential relationship between the ephrin B2/Dvl2 complex and the c-Jun/c-Fos/ Nfatcl complex. It is well characterized that downstream of Dvl, three main Wnt pathways can be activated $(35,36)$. Through the Wnt-signaling PCP pathway, Dvl can activate small Rho GTPases resulting from the activation of the c-Jun N-terminal kinase (JNK) pathway (37), which is also one of the downstream signaling pathways of RANKL-RANK signaling. In addition, Wnt proteins can signal through a calcium pathway that requires calcium mobilization and activation of PKC (37) modulated by Dvl. Nfatcl can also be activated through the $\mathrm{Ca}^{2+}$-dependent calcineurin pathway following RANKL-induced osteoclast differentiation (38). Collectively, we estimate that the ephrin B2/Dvl2 complex may be one of the positive elements that modulates the induction of c-Jun/c-Fos and their transcriptional target, Nfatc1, in the RANKL-induced osteoclastogenesis of RAW264.7 cells.

Finally, this study explored whether EphB4-ephrin B2 reverse signaling affected the expression level of Dvl2. Using Western blotting, we found that the bands for Dvl 2 in the EphB4-Fc-treated group and the blank control group (without EphB4-Fc, Fc or RANKL) were weaker than those in the Fc treatment group following RANKL-induced osteoclastogenesis (Fig. 6B). This indicated that after binding of EphB4, the downstream signal of ephrin B2 via Dvl2 inhibited RANKL-induced osteoclast differentiation of RAW264.7 cells and in turn, down-regulated the expression level of Dvl2 in a negative feedback mechanism.

It should be noted that this study has provided only biochemical proof for the interaction of Dvl2 and ephrin B2. However, we will perform gain-of-function and loss-of-function experiments to reveal the biological significance and the molecular mechanisms of Dvl2 in ephrin B2 reverse signaling. By proposing the participation of $\mathrm{Dvl} 2$ as a possible mediator downstream of the EphB4-ephrin B2 reverse signaling, we may provide a pharmacological target for modulating, at least in part, the coupling between osteoclasts and osteoblasts as a novel therapy for bone diseases. However, further studies are needed to explore this possibility. For example, what exact role does Dvl2 protein have downstream of EphB4/ephrin B2 reverse signaling? Are there any other molecules related to the ephrin B2/Dvl2 complex that interact with RANKL-RANK signaling? Collectively, our results indicate that Dvl2 could be the potential PDZ-domain protein acting downstream of ephrin B2 in the RANKL-induced osteoclast differentiation of RAW264.7 cells, providing a potential novel therapeutic target for bone diseases.

\section{Acknowledgements}

We thank the Zhejiang California International NanoSystems Institute (ZCNI) for technical support. This study was supported by a grant from the National Natural Science Foundation of China (30700958) and by the Zhejiang Provincial Natural Science Foundation of China (Y2100333).

\section{References}

1. Manolagas SC: Birth and death of bone cells: basic regulatory mechanisms and implications for the pathogenesis and treatment of osteoporosis. Endocr Rev 21: 115-137, 2000.

2. Mundy GR and Elefteriou F: Boning up on ephrin signaling. Cell 126: 441-443, 2006.

3. Teitelbaum SL and Ross FP: Genetic regulation of osteoclast development and function. Nat Rev Genet 4: 638-649, 2003.

4. Anderson DM, Maraskovsky E, Billingsley WL, et al: A homologue of the TNF receptor and its ligand enhance T-cell growth and dendritic-cell function. Nature 390: 175-179, 1997.

5. Wong BR, Rho J, Arron J, et al: TRANCE is a novel ligand of the tumor necrosis factor receptor family that activates c-Jun N-terminal kinase in T cells. J Biol Chem 272: 25190-25194, 1997.

6. Yasuda H, Shima N, Nakagawa N, et al: Osteoclast differentiation factor is a ligand for osteoprotegerin/osteoclastogenesis-inhibitory factor and is identical to TRANCE/RANKL. Proc Natl Acad Sci USA 95: 3597-3602, 1998.

7. Yasuda H, Shima N, Nakagawa N, et al: Identity of osteoclastogenesis inhibitory factor (OCIF) and osteoprotegerin (OPG): a mechanism by which OPG/OCIF inhibits osteoclastogenesis in vitro. Endocrinology 139: 1329-1337, 1998.

8. Tsuda E, Goto M, Mochizuki S, et al: Isolation of a novel cytokine from human fibroblasts that specifically inhibits osteoclastogenesis. Biochem Biophys Res Commun 234: 137-142, 1997.

9. Simonet WS, Lacey DL, Dunstan CR, et al: Osteoprotegerin: a novel secreted protein involved in the regulation of bone density. Cell 89: 309-319, 1997.

10. Teitelbaum SL: Bone resorption by osteoclasts. Science 289: 1504-1508, 2000.

11. Lam J, Takeshita S, Barker JE, Kanagawa O, Ross FP and Teitelbaum SL: TNF-alpha induces osteoclastogenesis by direct stimulation of macrophages exposed to permissive levels of RANK ligand. J Clin Invest 106: 1481-1488, 2000.

12. Zhao C, Irie N, Takada Y, et al: Bidirectional ephrin B2-EphB4 signaling controls bone homeostasis. Cell Metab 4: 111-121, 2006.

13. Pasquale EB: Eph-ephrin bidirectional signaling in physiology and disease. Cell 133: 38-52, 2008.

14. Kullander K and Klein R: Mechanisms and functions of Eph and ephrin signalling. Nat Rev Mol Cell Biol 3: 475-486, 2002.

15. Allan EH, Hausler KD, Wei T, et al: Ephrin B2 regulation by PTH and PTHrP revealed by molecular profiling in differentiating osteoblasts. J Bone Miner Res 23: 1170-1181, 2008.

16. Gale NW and Yancopoulos GD: Growth factors acting via endothelial cell-specific receptor tyrosine kinases: VEGFs, angiopoietins, and ephrins in vascular development. Genes Dev 13: 1055-1066, 1999.

17. Myshkin E and Wang B: Chemometrical classification of ephrin ligands and Eph kinases using GRID/CPCA approach. J Chem Inf Comput Sci 43: 1004-1010, 2003.

18. Rahman MM, Kukita A, Kukita T, Shobuike T, Nakamura T and Kohashi O: Two histone deacetylase inhibitors, trichostatin A and sodium butyrate, suppress differentiation into osteoclasts but not into macrophages. Blood 101: 3451-3459, 2003. 
19. Faccio R, Teitelbaum SL, Fujikawa K, et al: Vav3 regulates osteoclast function and bone mass. Nat Med 11: 284-290, 2005.

20. Takayanagi H, Kim S, Koga T, et al: Induction and activation of the transcription factor NFATc1 (NFAT2) integrate RANKL signaling in terminal differentiation of osteoclasts. Dev Cell 3: 889-901, 2002.

21. Wada T, Nakashima T, Hiroshi N and Penninger JM: RANKLRANK signaling in osteoclastogenesis and bone disease. Trends Mol Med 12: 17-25, 2006.

22. Asagiri M, Sato K, Usami T, et al: Autoamplification of NFATc1 expression determines its essential role in bone homeostasis. J Exp Med 202: 1261-1269, 2005.

23. Ikeda F, Nishimura R, Matsubara T, et al: Critical roles of c-Jun signaling in regulation of NFAT family and RANKL-regulated osteoclast differentiation. J Clin Invest 114: 475-484, 2004

24. Matsuo K, Galson DL, Zhao C, et al: Nuclear factor of activated T-cells (NFAT) rescues osteoclastogenesis in precursors lacking c-Fos. J Biol Chem 279: 26475-26480, 2004

25. Makinen T, Adams RH, Bailey J, et al: PDZ interaction site in ephrin B2 is required for the remodeling of lymphatic vasculature. Genes Dev 19: 397-410, 2005.

26. Arvanitis D and Davy A: Eph/ephrin signaling: networks. Genes Dev 22: 416-429, 2008

27. Cifuentes M, Fuentes C, Mattar P, et al: Obesity-associated proinflammatory cytokines increase calcium sensing receptor (CaSR) protein expression in primary human adipocytes and LS14 human adipose cell line. Arch Biochem Biophys 500: $151-156,2010$

28. Sanchez-Fernandez MA, Gallois A, Riedl T, Jurdic P and Hoflack B: Osteoclasts control osteoblast chemotaxis via PDGF-BB/PDGF receptor beta signaling. PLoS One 3: e3537, 2008.

29. Gaasch JA, Bolwahnn AB and Lindsey JS: Hepatocyte growth factor-regulated genes in differentiated RAW 264.7 osteoclast and undifferentiated cells. Gene 369: 142-152, 2006.
30. Rahman MM, Bhattacharya A and Fernandes G: Conjugated linoleic acid inhibits osteoclast differentiation of RAW264.7 cells by modulating RANKL signaling. J Lipid Res 47: 1739-1748, 2006.

31. Lee HS, Bong YS, Moore KB, Soria K, Moody SA and Daar IO: Dishevelled mediates ephrin B1 signalling in the eye field through the planar cell polarity pathway. Nat Cell Biol 8: 55-63, 2006.

32. Lee HS, Mood K, Battu G, Ji YJ, Singh A and Daar IO: Fibroblast growth factor receptor-induced phosphorylation of ephrin B1 modulates its interaction with Dishevelled. Mol Biol Cell 20: 124-133, 2009.

33. Tanaka M, Kamo T, Ota S and Sugimura H: Association of Dishevelled with Eph tyrosine kinase receptor and ephrin mediates cell repulsion. EMBO J 22: 847-858, 2003.

34. Davy A and Soriano P: Ephrin-B2 forward signaling regulates somite patterning and neural crest cell development. Dev Biol 304: 182-193, 2007.

35. Ciani L and Salinas PC: c-Jun N-terminal kinase (JNK) cooperates with Gsk3beta to regulate Dishevelled-mediated microtubule stability. BMC Cell Biol 8: 27, 2007.

36. Wechezak AR and Coan DE: Dvl2 silencing in postdevelopmental cells results in aberrant cell membrane activity and actin disorganization. J Cell Physiol 202: 867-873, 2005.

37. Wallingford JB and Habas R: The developmental biology of Dishevelled: an enigmatic protein governing cell fate and cell polarity. Development 132: 4421-4436, 2005.

38. Leibbrandt $A$ and Penninger JM: RANK/RANKL: regulators of immune responses and bone physiology. Ann NY Acad Sci 1143: $123-150,2008$ 